\title{
PET Imaging of Post-infarct Myocardial Inflammation
}

\author{
Andrej Ćorović ${ }^{1} \cdot$ Meritxell Nus $^{1} \cdot$ Ziad Mallat $^{1} \cdot$ James H. F. Rudd ${ }^{1} \cdot$ Jason M. Tarkin ${ }^{1}$
}

Accepted: 14 May 2021 / Published online: 1 July 2021

(C) The Author(s) 2021

\begin{abstract}
Purpose of Review To examine the use of positron emission tomography (PET) for imaging post-infarct myocardial inflammation and repair.

Recent Findings Dysregulated immune responses after myocardial infarction are associated with adverse cardiac remodelling and an increased likelihood of ischaemic heart failure. PET imaging utilising novel tracers can be applied to visualise different components of the post-infarction inflammatory and repair processes. This approach could offer unique pathophysiological insights that could prove useful for the identification and risk-stratification of individuals who would ultimately benefit most from emerging immune-modulating therapies. PET imaging could also bridge the clinical translational gap as a surrogate measure of drug efficacy in early-stage clinical trials in patients with myocardial infarction. The use of hybrid PET/MR imaging, in particular, offers the additional advantage of simultaneous in vivo molecular imaging and detailed assessment of myocardial function, viability and tissue characterisation.

Summary Further research is needed to realise the true clinical translational value of PET imaging after myocardial infarction.
\end{abstract}

Keywords PET $\cdot$ Myocardial infarction $\cdot$ Inflammation $\cdot$ Molecular imaging $\cdot$ Non-invasive imaging $\cdot$ Heart failure

\section{Introduction}

Myocardial infarction is a major global cause of morbidity and mortality, both due to acute complications arising at the time of initial presentation, as well as longer-term sequelae including chronic heart failure. After myocardial infarction, inflammation and its resolution play key roles in the promotion of

This article is part of the Topical Collection on Nuclear Cardiology

Jason M. Tarkin

jt545@cam.ac.uk

Andrej Ćorović

ac476@cam.ac.uk

Meritxell Nus

mn421@cam.ac.uk

Ziad Mallat

zm255@medschl.cam.ac.uk

James H. F. Rudd

jhfr2@cam.ac.uk

1 Division of Cardiovascular Medicine, University of Cambridge, Cambridge, UK healing responses and ultimately act to limit the degree of acute cardiac injury and the severity of subsequent adverse cardiac remodelling.

Indeed, inflammation is an automatic and necessary byproduct of ischaemic myocyte necrosis, which seeks to clear and organise the damaged tissue. A broadly biphasic immune response to myocardial infarction occurs, wherein an initial 'pro-inflammatory phase,' characterised by the sequential mobilisation of various pro-inflammatory cell types, is followed by a 'reparative phase' where the main role of the immune system is to facilitate wound healing, involving myofibroblast proliferation, neovascularisation, fibrosis and scar formation [1]. However, an excessive or unbalanced inflammatory response is associated with an increased risk of long-term heart failure [2].

In the light of the landmark CANTOS and COLCOT trials, which established the proof-of-principle that certain anti-inflammatory therapies can reduce cardiovascular events in patients with prior myocardial infarction and initial data indicating an additional signal for reduction in heart failure-related events, the tantalising prospect is thus presented that pharmacological manipulation of immune responses in the post-infarct period could be clinically beneficial $[3,4,5 \cdot \bullet]$. 
Given the variation in clinical presentations and the heterogeneity of inflammatory cell phenotypes at play within the infarcted heart, it is likely that targeted immunosuppressive therapies applied in specific high-risk patient populations would yield far greater overall benefit than more 'blanket' broad-spectrum approaches that have been trialled in the past [6].

In this regard, positron emission tomography (PET) imaging presents a unique opportunity to probe and visualise molecular mechanisms occurring after myocardial infarction in vivo, and to directly investigate the effects of novel therapies on myocardial inflammation. This article provides a focused review of recent progress in the use of PET imaging for imaging post-infarct myocardial inflammation and related processes.

\section{Cellular Biology of Post-infarct Inflammation}

An understanding of the cellular biology of post-infarct inflammation is important for contextualising the significance of cardiac PET signals, although detailed consideration of this topic is beyond the scope of this article. The reader is directed to more comprehensive reviews elsewhere $[1,7,8]$.

The cardiomyocyte necrosis that follows cardiac ischaemia sets in motion a chain of events closely linked to immune activation and resolution, which ultimately results in the formation of stable fibrotic scar tissue. However, if this balanced and coordinated immune response fails, uncontrolled initial or resurgent inflammation, fuelled by dysregulation of the mononuclear phagocytic network, can contribute to the risk of developing long-term heart failure [9].

In the initial pro-inflammatory phase, neutrophils are among the first leucocytes to migrate to the site of injury whereupon they phagocytose cellular debris, degrade extracellular matrix via the release of matrix metalloproteinases and generate reactive oxygen species. Monocytes are also recruited from the splenic reservoir and bone marrow via adrenergic signalling, whereupon they differentiate into tissue macrophages and likewise undertake phagocytosis, proteolysis and promote inflammation through generation of reactive oxygen species and secretion of pro-inflammatory cytokines (e.g. IL-1 $\beta$, IL-6 and TNF $\alpha$ ). Monocyte recruitment occurs as early as $30 \mathrm{~min}$ after the onset of ischaemia and up to $40 \%$ of the monocytes recruited within the first $24 \mathrm{~h}$ originate from the spleen $[10,11]$. B and T lymphocytes and natural killer cells are also crucial components of the initial and subsequent inflammatory responses. In particular, $\mathrm{T}$ regulatory cells help direct the transition from post-infarct inflammation towards resolution [12].

In mouse models, flow cytometry performed after coronary ligation has shown that pro-inflammatory Ly- $6 \mathrm{C}^{\text {hi }}$ monocytes (analogous to classical $\mathrm{CD} 14^{+} \mathrm{CD} 16^{-}$and intermediate
$\mathrm{CD} 14^{+} \mathrm{CD} 16^{+}$monocytes in humans), which have a life span of about $20 \mathrm{~h}$, predominate at days 1-4 post-infarction [13]. By day 5-14 post-infarction, through a combination of differentiation and switch from CCR2 to CXC3CL1-mediated recruitment, these cells are replaced by a predominance of Ly$6 \mathrm{C}^{\text {lo }}$ monocytes (analogous to non-classical $\mathrm{CD} 14^{\mathrm{int}} \mathrm{CD} 16^{+}$ monocytes in humans), which give rise to macrophages whose secretory cytokine profile includes IL-10, TGF $\beta$ and VEGF. These cytokines exert anti-inflammatory actions including downregulation of Th1 cell responses, and promotion of angiogenesis and fibrosis through interactions with endothelial cells and fibroblasts, respectively. This well-characterised transition in monocyte phenotype was also observed in a human post-mortem study [14]. This study showed increased pro-inflammatory $\mathrm{CD} 14^{+} \mathrm{CD} 16^{-}$cells in the infarct border zone during the acute inflammatory phase, and comparable numbers of $\mathrm{CD}_{1} 4^{+} \mathrm{CD} 16^{-}$and non-classical $\mathrm{CD} 14^{+} \mathrm{CD} 16^{+}$ cells in the infarct core at later time points, which coincided with depletion of splenic monocytes. Although several studies have shown different correlations with peripheral blood monocytes in patients with myocardial infarction, the role of non-classical monocytes in this context remains largely unknown.

In another study of 36 patients, increased peak levels of peripheral $\mathrm{CD} 14^{+} \mathrm{CD} 16^{-}$monocytes measured after acute myocardial infarction were negatively associated with the likelihood of longer-term left ventricular functional recovery [15]. Increased leukocyte concentration was also associated with a greater incidence of heart failure hospitalisations in a study of 16,940 men from the general population without prior history of myocardial infarction or stroke enrolled in a cardiovascular screening programme in Sweden [16]. In mice, attenuation of monocyte recruitment to the ischaemic myocardium achieved by $\mathrm{B}$ cell depletion and silencing of endothelial cell adhesion molecules or the monocyte chemokine receptor CCR2 were, conversely, associated with preserved ejection fraction post-infarct [17-19]. The beneficial actions of angiotensin-converting enzyme inhibition on left ventricular function after myocardial infarction may also in part be related to reduction in recruitment of splenic-derived monocytes to the healing myocardium [20].

Divergent subsets of cardiac CCR2 ${ }^{+}$and $\mathrm{CCR} 2^{-}$macrophages have also been described [21]. While monocytederived cardiac $\mathrm{CCR} 2^{+}$macrophages are pro-inflammatory, tissue-resident $\mathrm{CCR} 2^{-}$macrophages have non-redundant cardioprotective roles in tissue remodelling and cardiac regeneration, and largely self-renew via local proliferation [22]. During post-myocardial infarction remodelling, increases in cardiac macrophages occurring in the remote myocardium are due to local proliferation and to a lesser extent monocyte recruitment [18].

Lower numbers of $\mathrm{CCR} 2^{+}$macrophages have been associated with improvement in left ventricular systolic function in 
patients with severe heart failure undergoing left ventricular assist device implantation, as has also been shown in mice [23, 24]. In other murine studies, directing cardiac macrophage populations towards a reparative phenotype using either small interfering RNA directed at the transcription factor IRF5, or subcutaneous IL-10 injection, led to improvements in left ventricular function post infarct $[25,26]$.

Traditionally, the functions of the broadly divergent macrophage types in the post-infarct myocardium have been ascribed to 'M1-like' and 'M2-like' macrophages. However, it is important to note that this classification system is based on a spectrum of in vitro activation profiles rather than the broader transcriptional repertoire of macrophages that is now known to exist [27]. There is also incomplete overlap with M1 and $\mathrm{M} 2$ markers in cardiac $\mathrm{CCR} 2^{+} / \mathrm{CCR} 2^{-}$macrophages [23]. Indeed, data from studies that have applied unbiased highthroughput sequencing techniques have revealed a widerange of both resident and monocyte-derived macrophage sub-types that co-exist in the infarct, peri-infarct and remote myocardium [28]. The occurrence of macrophage plasticity and the role of metabolic and epigenetic reprogramming of multi-level systemic immune cell activation add to the level of complexity [29].

Apoptosis and the clearance of apoptotic cells (efferocytosis) are another important component of postinfarction damage resolution, and experimental suppression of efferocytosis has been shown to result in larger myocardial infarct size and greater systolic dysfunction [30].

Because these key inflammatory processes are vital for determining the extent of myocardial damage and likelihood of long-term heart failure after myocardial infarction, there is a role for molecular imaging techniques such as PET to study these disease mechanisms in efforts to inform the design and use of new therapeutic strategies for ischaemic heart failure.

\section{PET Tracers for Imaging Post-infarct Inflammation}

Molecular imaging of myocardial inflammation with PET offers the advantage of enabling the in situ visualisation, in realtime, of post-infarction processes. Moreover, by utilising novel PET tracers that are specific for particular inflammatory cell types or processes, individual components of the initial inflammatory and subsequent healing responses can potentially be interrogated.

\section{${ }^{18}$ F-FDG Imaging After Myocardial Infarction}

${ }^{18} \mathrm{~F}$-fluorodeoxyglucose $\left({ }^{18} \mathrm{~F}\right.$-FDG $)$ is the most commonly used PET tracer for inflammation imaging in clinical medicine, and several studies of post-infarct imaging testify to the general feasibility of this imaging approach.
Using PET/CT in a mouse model of myocardial infarction, one study showed increased ${ }^{18} \mathrm{~F}$-FDG uptake within infarcted myocardium on day 5 post-infarct compared to control animals, which co-localised with delayed gadolinium enhancement on MRI [31]. The highest ${ }^{18}$ F-FDG signals were observed at the infarct border zone, which corresponded with the site of peak monocyte/macrophage recruitment in some cases. Increased tracer uptake was also confirmed in monocytes/macrophages isolated from the infarcted mouse hearts, compared to the surrounding infarct tissue.

Similar findings for ${ }^{18} \mathrm{~F}$-FDG have been observed in several clinical studies. For example, one study including 15 patients imaged within 7 days of a first myocardial infarction showed the metabolic rate of ${ }^{18} \mathrm{~F}$-FDG uptake to be increased in infarcted versus remote myocardium [32]. The PET signal was higher in myocardial segments with late gadolinium enhancement and oedema, versus oedema alone in this study. In 5 control patients with previous stable myocardial infarction, a reverse pattern was observed, with lower ${ }^{18} \mathrm{~F}$-FDG uptake in the chronic infarct compared to remote zone. Another study that analysed 39 patients with ST-segment elevation myocardial infarction found that elevated infarct-related ${ }^{18} \mathrm{~F}$-FDG uptake in the first week after myocardial infarction was associated with an increased likelihood of left ventricular impairment on MRI after 6 months, independent of infarct size [33•]. In this study, ${ }^{18} \mathrm{~F}-\mathrm{FDG}$ uptake in the infarcted myocardium was also correlated with ${ }^{99 \mathrm{~m}} \mathrm{Tc}$-sestamibi SPECTdefined area at risk and peripheral blood counts of $\mathrm{CD} 14^{\text {high }} \mathrm{CD} 16^{+}$monocytes.

However, despite these encouraging data, myocardial imaging using ${ }^{18} \mathrm{~F}-\mathrm{FDG}$ is nonetheless challenging due to avid physiological background uptake. Pre-scan patient preparation for cardiac ${ }^{18} \mathrm{~F}-\mathrm{FDG}$ imaging requires cumbersome myocardial suppression protocols, which are often ineffective. The effect of dietary manipulation on the already altered metabolic state of viable, post-ischaemic cardiac myocytes is also unknown. Moreover, while ${ }^{18} \mathrm{~F}$-FDG is a clinically useful measure of metabolic activity in inflammatory cells such as macrophages in several cardiovascular diseases, its uptake is not cell-specific.

To overcome these limitations, several novel PET tracers that could potentially provide more specific markers of inflammation and/or related cellular processes than ${ }^{18} \mathrm{~F}-\mathrm{FDG}$ have been investigated for use in myocardial imaging (Fig. 1 and Table 1).

\section{Tracers for Imaging Post-infarct Inflammation}

Aside from ${ }^{18} \mathrm{~F}$-FDG, other PET tracers that have been investigated for post-infarct myocardial inflammation imaging either bind cell-surface receptors that are known to be expressed on macrophages and other inflammatory cells, or are more selectively taken up by metabolically active inflammatory 


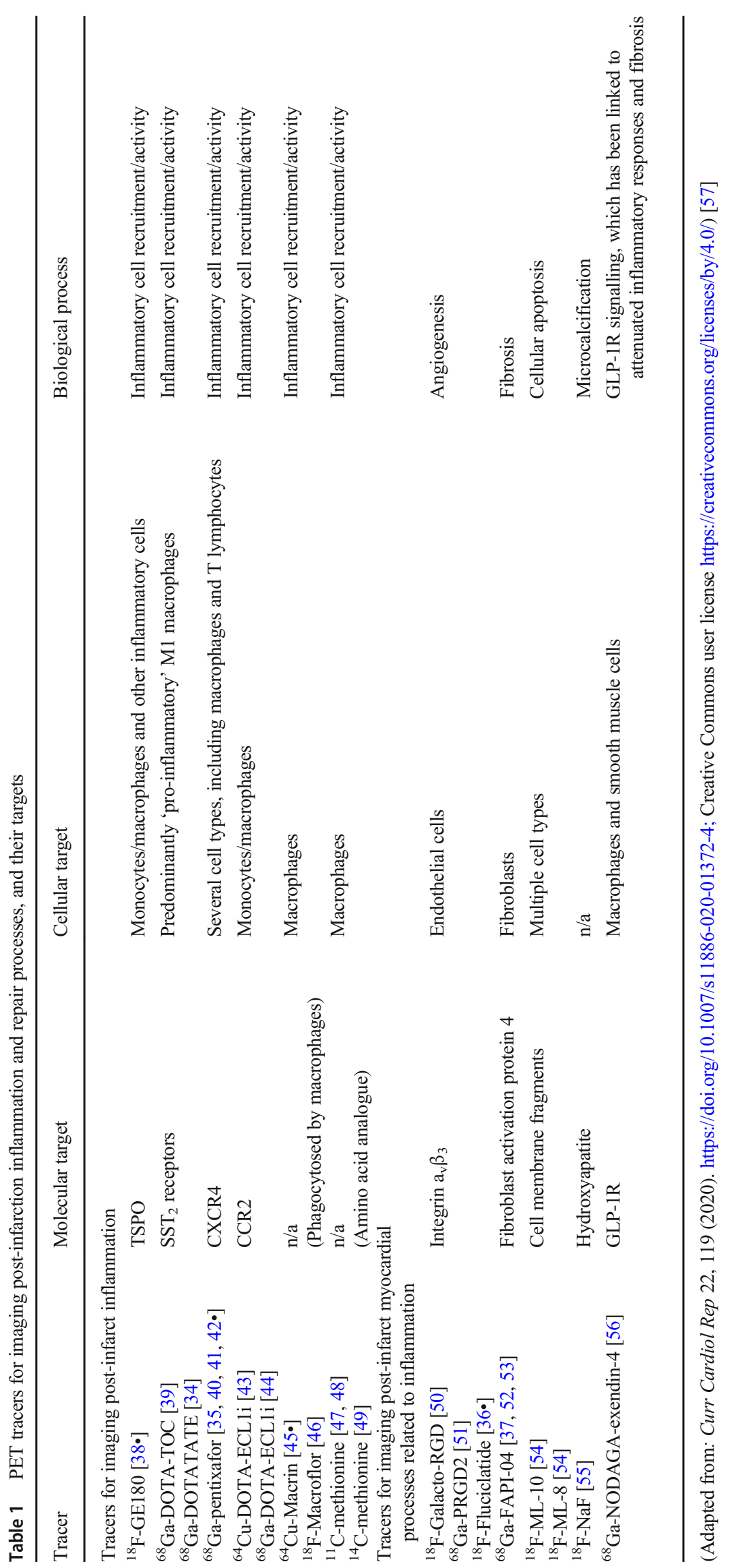



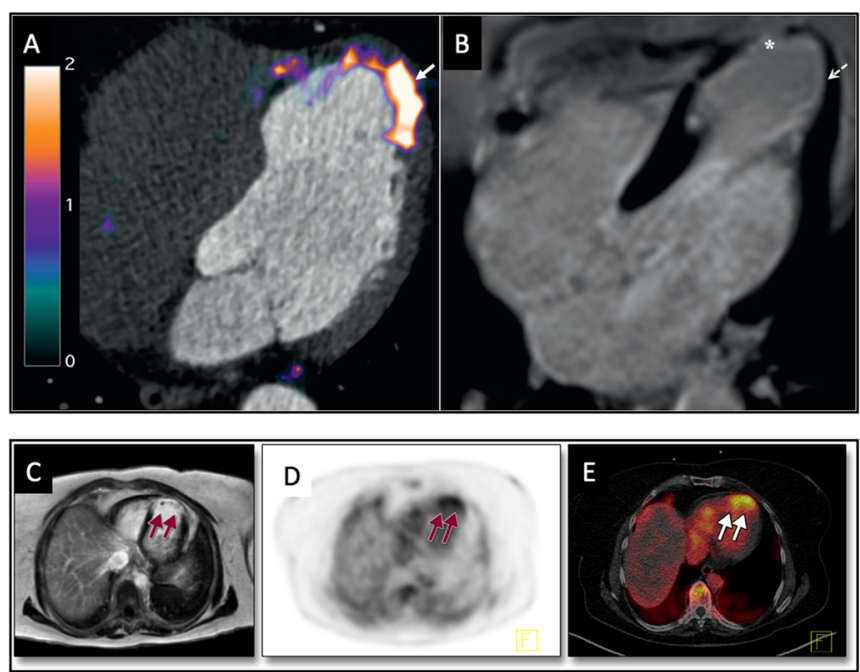

Fig. 1 PET imaging of inflammation and related processes after myocardial infarction. Left panels: tracers for imaging inflammatory cells. Top A ${ }^{68} \mathrm{Ga}$-DOTATATE ( $\mathrm{SST}_{2}$ ) PET-CT image demonstrating residual inflammation (arrow) in $\mathbf{B}$ partially viable myocardium with subendocardial infarct (dashed arrow), bordering full thickness scarring (asterisk) confirmed by late gadolinium enhancement magnetic resonance imaging, 4 years after a left anterior descending artery MI. (Reproduced from: $J$ Am Coll Cardiol. 2019;73:2489-91; doi: 10.1016/ j.jacc.2019.02.052; Creative Commons user license https:// creativecommons.org/licenses/by/4.0/) [34]. Bottom An apical myocardial infarct (arrows), visualised using contrast-enhanced multishot inversion recovery turbo field echo cardiac MRI $(\mathbf{C}),{ }^{68} \mathrm{Ga}-$ pentixafor (CXCR4) PET (D) and fused PET-CT (E). (Reprinted from

cells than ${ }^{18}$ F-FDG. Importantly, these tracers exhibit lower background myocardial activity than ${ }^{18} \mathrm{~F}-\mathrm{FDG}$, offering the potential for better differentiation of focal myocardial signals without the need for dietary myocardial suppression. While much of the key research in the field has so far been conducted in preclinical animal models, a summary of novel PET tracers applied in clinical studies is shown in Table 2.

Translocator Protein Receptor The translocator protein (TSPO) receptor is situated in the outer mitochondrial membrane and is expressed in activated mononuclear cells and other inflammatory cells. In a translational study, PET imaging with the TSPO tracer ${ }^{18} \mathrm{~F}$-GE180 revealed elevated myocardial TSPO signals at 1 week post-coronary artery ligation in mice, which co-localised to $\mathrm{CD}^{+} 8^{+}$inflammatory macrophages in the infarct [38•]. Early increases in TSPO PET signals after the infarct were predictive of subsequent adverse left ventricular remodelling at 8 weeks. In vitro data also demonstrated that M1 macrophages exhibited 7-fold higher TSPO uptake compared to M2 macrophages. Treatment of mice with an angiotensin-converting enzyme inhibitor also resulted in lower TSPO signal in the infarct compared to control animals. In the same study, feasibility of clinical imaging was shown in 3 patients with myocardial infarction who were found to have elevated infarct-related TSPO signal compared with healthy controls. Interestingly, changes in neuroinflammation
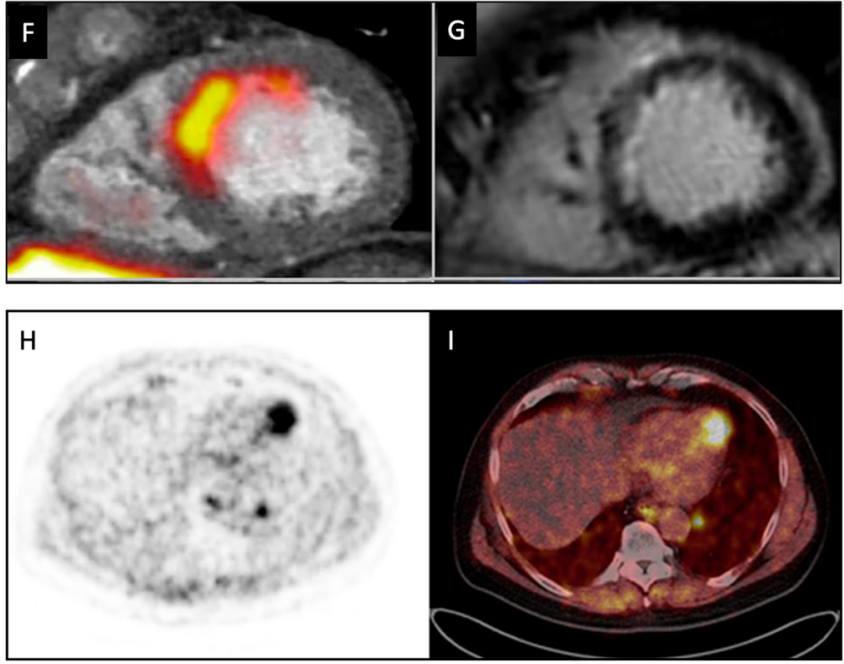

JACC Cardiovasc Imaging. 2015;8:1466-8, with permission from Elsevier) [35]. Right panels: tracers for imaging post-infarct myocardial processes related to inflammation. Top ${ }^{18} \mathrm{~F}$-Fluciclatide (angiogenesis) PET-CT (F) and MRI images $(\mathbf{G})$ of an antero-septal infarct. (Reproduced from: Heart. 2016;103:607-15; doi: 10.1136/heartjnl2016-310115; Creative Commons user license https://creativecommons. org/licenses/by/4.0/) [36•]. Bottom ${ }^{68}$ Ga-FAPI (fibrosis) imaging. In this patient with thyroid cancer, whole-body PET-CT imaging reveals ${ }^{68} \mathrm{Ga}$ FAPI uptake in the left ventricle as shown on the PET $(\mathbf{H})$ and fused PETCT (I) images. (Reproduced from: J Nucl Cardiol. 2020;1-10; doi: 10. 1007/s12350-020-02307-w; Creative Commons user license https:// creativecommons.org/licenses/by/4.0/) [37]

detected by TSPO occurred in parallel to changes in infarctrelated myocardial signals in both mice and patients.

Somatostatin Receptor Subtype-2 Upregulation of somatostatin receptor subtype-2 ( $\left.\mathrm{SST}_{2}\right)$ occurs in activated macrophages, offering another novel inflammation imaging target that may be useful in range of cardiovascular diseases $[58,59]$. Among the clinically available somatostatin receptor PET tracers, ${ }^{68} \mathrm{Ga}$-DOTATATE has the highest binding affinity for $\mathrm{SST}_{2}$. Although one preclinical study observed rapid blood clearance of ${ }^{68} \mathrm{Ga}$-DOTATATE following injection with no myocardial binding after coronary ligation in mice, initial clinical data appears more promising for this approach. In a study that included 6 patients with myocardial infarction who were imaged using the somatostatin receptor PET tracer ${ }^{68} \mathrm{Ga}$-DOTATOC within 3 to 10 days of symptoms, focal myocardial signals were observed in all patients [39]. Overall, there was good concordance between ${ }^{68} \mathrm{Ga}$ DOTATOC signal and infarcted myocardial segments with late gadolinium enhancement and oedema on MRI. In an exploratory post hoc analysis of 12 patients with myocardial infarction who underwent prospective cardiovascular ${ }^{68} \mathrm{Ga}-$ DOTATATE PET imaging, infarct-related myocardial $\mathrm{SST}_{2}$ signals were increased in patients with recent myocardial infarction [34] (Fig. 1). In this study, focal myocardial ${ }^{68} \mathrm{Ga}-$ DOTATATE signals were observed in damaged myocardial 


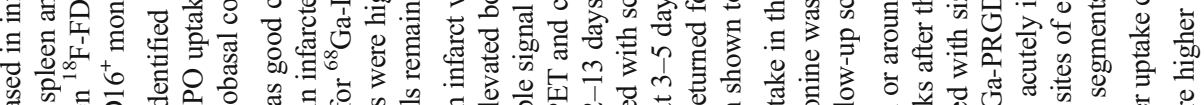

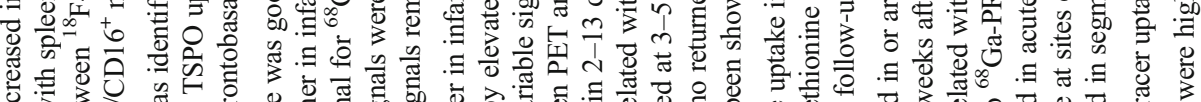

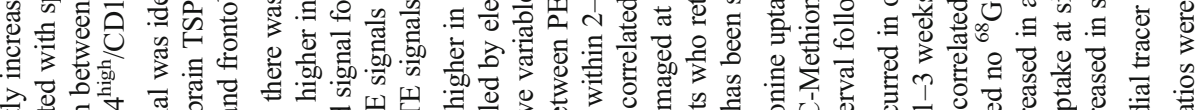

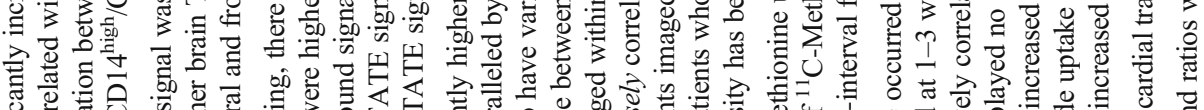

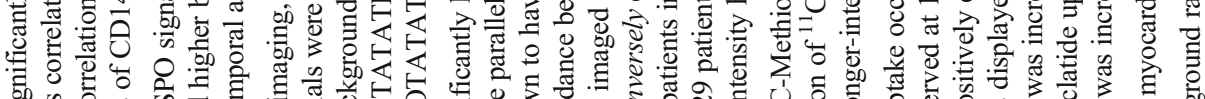

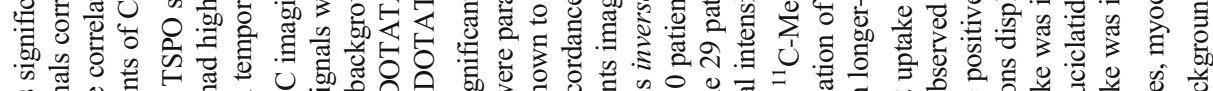

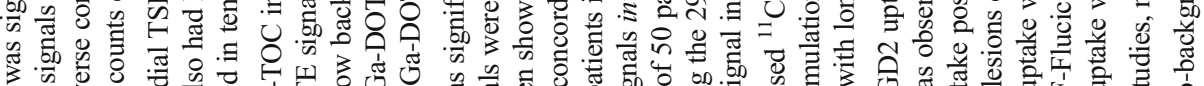

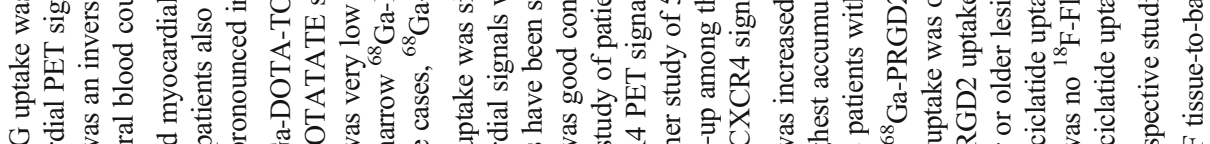

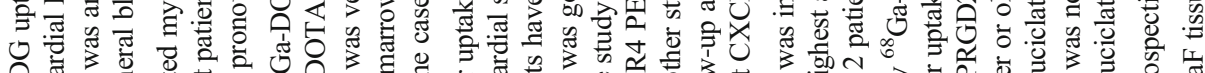

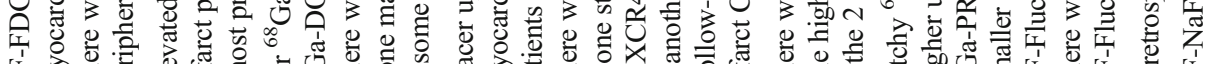

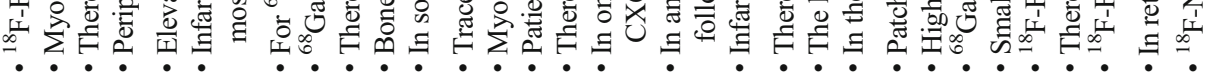

\section{论}
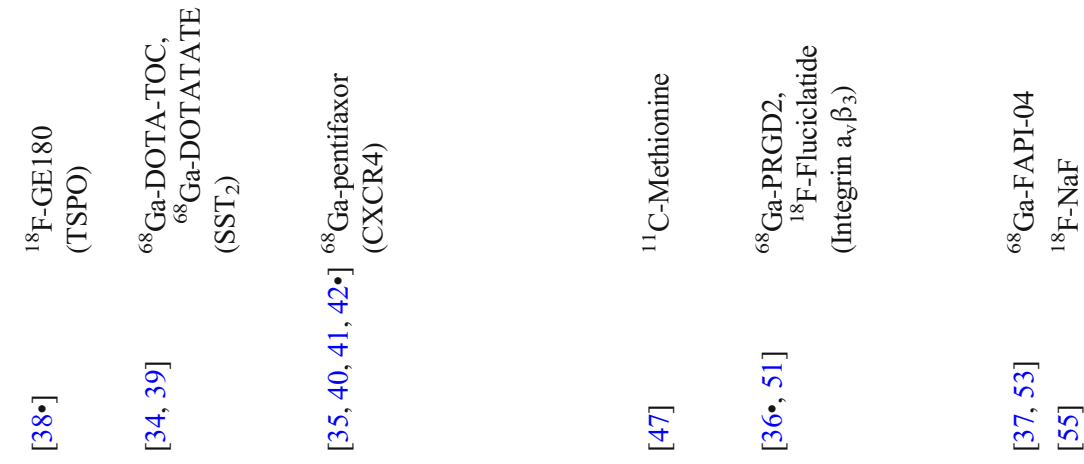

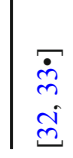


segments with echocardiographic regional wall motion abnormalities in patients with old myocardial infarction. Infarctrelated ${ }^{68} \mathrm{Ga}$-DOTATATE signals were also correlated with increased metabolic bone marrow activity measured by ${ }^{18} \mathrm{~F}$ FDG PET. A pre-specified sub-study of an ongoing prospective observational study aiming to characterise the natural history of coronary and myocardial ${ }^{68} \mathrm{Ga}$-DOTATATE inflammatory signals after myocardial infarction (ClinicalTrials.gov Identifier: NCT04073810) will shed further light on these initial observations.

C-X-C Chemokine Receptor Type-4 The C-X-C motif chemokine receptor type 4 (CXCR4) is expressed on the surface of several inflammatory cell types, including macrophages, neutrophils and lymphocytes. In one study, uptake of the CXCR4-targeted tracer ${ }^{68} \mathrm{Ga}$-pentixafor peaked in the infarct region 3 days post-myocardial infarction in mice and corresponded with a flow cytometry-based peak of circulating $\mathrm{CD} 45^{+}$leukocytes and histological staining of macrophages and granulocytes within the infarct [40]. As with the TSPO ligand, CXCR4 PET signals in mouse infarcts were also attenuated by pre-administration of enalapril. As part of the same study, feasibility of clinical imaging was shown in 12 patients who underwent ${ }^{68} \mathrm{Ga}$-pentixafor PET/CT and cardiac MRI within 8 days of an ST-segment elevation myocardial infarction. In these patients, CXCR4 PET signals were higher in the infarct region than remote myocardium and were correlated with CXCR4 signals in the bone marrow and spleen.

In a small clinical study of 7 patients with myocardial infarction who underwent ${ }^{68} \mathrm{Ga}$-pentixafor PET imaging within 5-10 days of the event, only 3 of the 7 patients showed focal uptake in the infarct as defined by MRI [35] (Fig. 1). Another study showed infarct-related ${ }^{68}$ Ga-pentixafor uptake in 17 of 22 patients imaged within the first 2 weeks after the event [41]. In this study, myocardial CXCR4 signals were inversely associated with time from symptoms to scanning and were also negatively associated with scar volume assessed by late gadolinium MRI at a median 4-month follow-up. Residual myocardial ${ }^{68} \mathrm{Ga}$-pentixafor uptake at this late timepoint could represent leucocyte involvement in the healing process, but without direct histological comparison, this notion remains speculative.

In another translational study involving 180 mice, persistent myocardial ${ }^{68} \mathrm{Ga}$-pentixafor PET signals occurring beyond 3 days after coronary ligation were observed more often in animals that died of left ventricular rupture than those who survived and were associated with worse cardiac function at 6 weeks [42•]. Moreover, the incidence of left ventricular rupture was reduced by administration of a CXCR4 blocking agent within the first 3 days post-MI. When compared to histology, myocardial ${ }^{68}$ Ga-pentixafor uptake co-localised with CD68 ${ }^{+}$inflammatory cells and was also associated with increased numbers of both Ly6 $\mathrm{C}^{\text {high }}$ and Ly6 $\mathrm{C}^{\text {low }}$ peripheral monocyte counts. These findings were confirmed in $50 \mathrm{pa}-$ tients with myocardial infarction. In these patients, CXCR4 signals showed a weak-to-moderate inverse correlation with left ventricular function at baseline and after follow-up of approximately 7 months.

Another study showed increased ${ }^{68} \mathrm{Ga}$-pentixafor uptake in mediastinal lymph nodes in patients after myocardial infarction because of $T$ cell accumulation [60]. The findings of this study support a potential role for CXCR4 blockade postinfarction as a means of mobilising T-regulatory cells to the injured myocardium, which have been shown to limit adverse cardiac remodelling in pre-clinical models [61].

Chemokine Receptor Type-2 C-C chemokine receptor type 2 (CCR2) is another important inflammatory cell marker involved in monocyte/macrophage recruitment, which has been evaluated for imaging myocardial inflammation using PET. In an ischaemia-reperfusion heterotopic heart transplantation mouse model, the PET tracer ${ }^{64} \mathrm{Cu}$-DOTA-ECL1i was shown to bind $\mathrm{CCR} 2^{+}$monocytes and macrophages within donor hearts, demonstrating increased tracer uptake compared to native hearts [43]. Specific autoradiographic binding was also confirmed in human myocardial specimens. Comparable myocardial PET signal intensity was observed with the ${ }^{64} \mathrm{Cu}$-labelled tracer to data from a previous study on ${ }^{68} \mathrm{Ga}$ DOTA-ECL1i [44].

$\mathrm{As} \mathrm{CCR2}^{+}$macrophage depletion has demonstrated beneficial effects on cardiac function post-infarction in mouse models, CCR2-targeted PET tracers could also be useful for the clinical evaluation of CCR2 inhibitors in future early-stage clinical trials [24].

Macrophage Phagocytosis ${ }^{64} \mathrm{Cu}$-Macrin is a 20 -nm spherical dextran nanoparticle tracer designed specifically to target macrophages. In a preclinical study, ${ }^{64} \mathrm{Cu}$-Macrin was shown to report on the accumulation of macrophages within the infarcted myocardium in experiments using mice, rabbits and pigs [45•]. Increases in lung and cardiac ${ }^{64} \mathrm{Cu}$-Macrin PET signals were also observed in mouse models of sepsis and pneumonia, in newborn mice exhibiting higher macrophage content than adults, and in atherosclerotic plaques in rabbits. Comparison of a near-infrared fluorescent version of the nanoparticle with flow cytometry and confocal staining showed that the tracer uptake occurred predominately in $\mathrm{MHCII}^{\text {high }}$ $\mathrm{CCR} 2{ }^{\text {high }}$ tissue macrophages. In contrast, ${ }^{64} \mathrm{Cu}$-Macrin uptake was negligible in neutrophils, fibroblasts and endothelial cells.

Amino Acid Metabolism Radio-labelled amino acid analogues represent another potential approach for imaging the metabolic activity of inflammatory cells after myocardial infarction. For example, the PET tracer ${ }^{11} \mathrm{C}$-methionine is thought to accumulate in macrophages, but not 
healthy myocytes. In a small clinical study, ${ }^{11} \mathrm{C}$-methionine PET was performed in 9 patients with left anterior descending artery myocardial infarctions treated by percutaneous coronary intervention, within 2 weeks of the event [47]. In these patients, increased ${ }^{11} \mathrm{C}$-methionine signal was observed in areas of low or absent ${ }^{201}$ Thallium SPECT and ${ }^{18} \mathrm{~F}$-FDG PET signal in the myocardium indicating tissue infarction, and subsequent reductions in ${ }^{11} \mathrm{C}$ methionine signals were seen in 2 patients imaged at 3 or 6 months. In another study, ${ }^{11} \mathrm{C}$-methionine PET imaging performed in mice showed increased tracer uptake in the infarcted myocardium at day 3 post-coronary ligation, compared to the remote myocardium and healthy control animals, which was associated with macrophage infiltration on histology [48]. The ${ }^{11} \mathrm{C}$-methionine PET signal declined by day 7 and was also inhibited by treatment with anti-integrin antibodies that lowered macrophage content. In the same study, ${ }^{11} \mathrm{C}$-methionine uptake was shown to occur more in M1 than M2 polarised macrophages. Further spatiotemporal profiling of infarct-related ${ }^{14} \mathrm{C}$-methionine signal was investigated in a rat model, which showed peak tracer uptake occurring at days 3 to 7 post-infarct, with a gradual decline until day 28 [49]. Lower tracer uptake occurring at later time points was felt to reflect myofibroblast activity or neoangiogenesis, rather than acute inflammation per se.

\section{Tracers for Imaging Post-infarct Myocardial Processes Related to Inflammation}

Neoangiogenesis Integrin $a_{v} \beta_{3}$ expressed by endothelial cells (as well as macrophages and other cells) has been examined for use as a PET imaging target for detecting neoangiogenesis. In one study, myocardial accumulation of the $\mathrm{a}_{\mathrm{v}} \beta_{3}$-targeted PET tracer ${ }^{18} \mathrm{~F}$-Galacto-RGD was observed from 3 days post-infarction in rats, with peak tracer uptake in the infarct between 1 and 3 weeks [50]. $a_{v} \beta_{3}$ PET signal intensity was associated with increased histological vascular density in post-infarct tissue specimens. In a clinical study, increased myocardial $a_{v} \beta_{3}$ PET signal was also observed using ${ }^{68} \mathrm{Ga}$-PRGD2 in 20 out of 23 patients imaged within 1 week after their event [51]. Another clinical study showed that ${ }^{18}$ F-Fluciclatide, which also binds $a_{v} \beta_{3}$, showed higher PET signals at the site of acute infarction in patients within 2 weeks of their event, compared to remote myocardium and patients with old infarction or healthy volunteers [36•] (Fig. 1). In this study, baseline ${ }^{18} \mathrm{~F}$ Fluciclatide uptake was also associated with an increased probability of subsequent functional left ventricular recovery after 9 months.

Cardiac Fibrosis Experimental tracers have been tested for imaging myofibroblast activity after myocardial infarction, which bind fibroblast activation protein. In one study performed in a rat model of infarction, ${ }^{68} \mathrm{Ga}$-FAPI-04 PET signals occurring at day 6 post-infarction were highest at the infarct border zone, which co-localised with histological myofibroblast staining [52]. A retrospective analysis of clinical images from 32 patients who underwent prior ${ }^{68} \mathrm{Ga}$-FAPI PET imaging for cancer staging showed focal myocardial ${ }^{68} \mathrm{Ga}$-FAPI uptake in patients with previous history of coronary artery disease and impaired left ventricular function [37] (Fig. 1). A similar association between focal cardiac ${ }^{68} \mathrm{Ga}-$ FAPI PET uptake and cardiovascular risk factors was found in another retrospective study of 229 patients with cancer [53].

Apoptosis and Microcalcification Other pathogenic processes occurring after myocardial infarction that have been targeted for molecular imaging with PET tracers include apoptosis and microcalcification. Indeed, given the importance of efferocytosis on post-infarct repair and inflammation resolution, detection of defective or delayed clearance of apoptotic cells by PET imaging could represent an important imaging target. A study of the novel apoptosis tracers ${ }^{18} \mathrm{~F}-\mathrm{ML}-10$ and ${ }^{18} \mathrm{~F}-\mathrm{ML}-8$ demonstrated focal infarct-related myocardial tracer accumulation on days 1 to 3 after coronary ligation in rats in regions devoid of ${ }^{18} \mathrm{~F}$-FDG uptake and histologically confirmed apoptosis [54]. In this study, ex vivo binding assays showed that ${ }^{18} \mathrm{~F}-\mathrm{ML}-8$ was taken up by apoptotic, but not necrotic or normal cells, presenting a potential advantage over Annexin V and synaptotagmin-I targeted SPECT tracers, which bind exposed phosphatidylserine in both apoptotic and necrotic cells.

Microcalcification is also thought to occur as part of the healing process after myocardial infarction. In one study of 10 patients, signal from the PET tracer ${ }^{18} \mathrm{~F}-\mathrm{NaF}$, which binds to areas of microcalcification and exposed hydroxyapatite in atherosclerosis, was increased more in areas of myocardial infarction with scar than the remote myocardium [55]. However, areas of increased myocardial ${ }^{18} \mathrm{~F}-\mathrm{NaF}$ PET signals occurring 1 day after myocardial infarction in rats were found to closely match histological markers of apoptosis, but not calcification.

Other Molecular Targets Among the other molecular targets that have been examined for post-myocardial infarction PET imaging is glucagon-like peptide-1 (GLP-1) [56]. This target is of particular interest as inhibition of the GLP1 receptor has been associated with improved cardiovascular outcomes in multiple clinical trials of patients with diabetes mellitus, although its effects on myocardial function are less clear [62]. Novel tracers for myocardial perfusion/infarct imaging, such as ${ }^{68} \mathrm{Ga}$-DOTA chelate, and for imaging myocardial oxidative metabolism, such as ${ }^{11} \mathrm{C}$-acetate, have also been investigated in preclinical studies and could be useful in this setting [63, 64]. 


\section{Future Directions}

Among the potential clinical translational applications of postinfarct myocardial PET imaging are as follows: (i) to identify and risk-stratify patients with maladaptive initial or 'residual' inflammatory responses contributing an increased likelihood of longterm heart failure, and (ii) to inform the design and use of emerging immune-modulatory therapies for cardiovascular disease. This type of mechanistic research may also yield valid, nonPET biomarkers that can be more easily rolled out in the clinic.

Several preclinical studies have shown that PET imaging, for example with tracers targeting TSPO or CXCR4, can quantify changes in myocardial inflammation after treatment with conventional heart failure therapies, such as angiotensin-converting enzyme inhibitors and angiotensin receptor blockers [38 $, 40,65$, 66]. In another study, treatment with a novel NOD-like receptor protein 3 inflammasome inhibitor MCC950 in mice reduced infarct-related ${ }^{18} \mathrm{~F}$-FDG inflammatory signals, along with histological grading of M1 macrophage and neutrophil infiltration, and improved myocardial viability after myocardial infarction [67]. Results of future clinical trials using PET imaging to examine the effects of novel therapies for modulating ischaemic myocardial inflammation are awaited.

Multi-tracer studies can potentially help further tease out individual inflammatory pathways or healing responses to improve the precision of therapeutic targeting after myocardial infarction. For example, a study published as an abstract showed that peripheral macrophage depletion in mice resulted in reduced ${ }^{18} \mathrm{~F}$ GE180 infarct-related uptake at day 7 compared to control myocardial infarction due to lower $\mathrm{CD} 68^{+}$macrophage content, but increased ${ }^{68} \mathrm{Ga}$-pentixafor signal because of sustained recruitment of $\mathrm{Ly} 6 \mathrm{G}^{+}$neutrophils, as well as active calcification of intracavity thrombus detected by ${ }^{18} \mathrm{Na}-\mathrm{NaF}[68]$.

Moreover, the use of multi-modality multi-parametric imaging, and in particular hybrid PET/MRI, offers the ability to combine molecular imaging of inflammation and adjunct processes with detailed tissue characterisation. The potential value of this combined approach was shown in a study of 25 patients who underwent hybrid ${ }^{18} \mathrm{~F}$-FDG PET/MRI within 5 days of revascularisation for myocardial infarction [69]. This study found that although changes in ${ }^{18} \mathrm{~F}-\mathrm{FDG}$ uptake, T1 mapping and extracellular volume tended to overlap anatomically within the infarcts of individual patients, a lack of quantitative correlation meant that these measures were reporting on different underlying biological processes or tissue properties. In another preclinical study, dual-imaging with ${ }^{18} \mathrm{~F}$-Macroflor PET (a dextran nanoparticle similar to ${ }^{18} \mathrm{~F}-$ Macrin) and MRI with MPO-Gd, which detects myeloperoxidase activity in neutrophils and Ly-6 $\mathrm{C}^{\text {high }}$ monocytes, was performed at day 2 and 6 post-infarct in mice. This study showed divergent temporal changes in PET and MRI signals in keeping with the well-characterised biphasic monocyte/macrophage phenotypic response after myocardial infarction [46].

\section{Conclusions}

Dysregulated post-infarction inflammation and remodelling confer adverse outcomes in patients after myocardial infarction. PET imaging of these processes can potentially be utilised in both the development and deployment of novel immunomodulatory therapies for this indication. Further research efforts should be directed towards realising the full potential of these novel molecular imaging techniques.

Funding A.Ć. is supported by a Cambridge British Heart Foundation (BHF) Centre of Research Excellence (CRE)-funded Clinical Research Training Fellowship. M.N. is supported by a BHF Intermediate Basic Science Fellowship (FS/20/23/34784). J.H.F.R is part-supported by the NIHR Cambridge Biomedical Research Centre, the BHF, HEFCE, the EPSRC and the Wellcome Trust. J.M.T is supported by the Wellcome Trust (211100/Z/18/Z), the BHF and the NIHR Imperial Biomedical Research Centre (BRC). This work was supported by the Cambridge BHF CRE (RE/18/1/34212).

\section{Declarations}

Conflict of Interest The authors declare that they have no conflict of interest.

Human and Animal Rights and Informed Consent This is a review article, which references papers that have used animal and/or human participants in their studies.

Open Access This article is licensed under a Creative Commons Attribution 4.0 International License, which permits use, sharing, adaptation, distribution and reproduction in any medium or format, as long as you give appropriate credit to the original author(s) and the source, provide a link to the Creative Commons licence, and indicate if changes were made. The images or other third party material in this article are included in the article's Creative Commons licence, unless indicated otherwise in a credit line to the material. If material is not included in the article's Creative Commons licence and your intended use is not permitted by statutory regulation or exceeds the permitted use, you will need to obtain permission directly from the copyright holder. To view a copy of this licence, visit http://creativecommons.org/licenses/by/4.0/.

\section{References}

Papers of particular interest, published recently, have been highlighted as:

- Of importance

•- Of major importance

1. Prabhu SD, Frangogiannis NG. The biological basis for cardiac repair after myocardial infarction. Circ Res. 2016;119:91-112.

2. Adamo L, Rocha-Resende C, Prabhu SD, Mann DL. Reappraising the role of inflammation in heart failure. Nat Rev Cardiol. 2020;17(5): 269-85.

3. Ridker PM, Everett BM, Thuren T, MacFadyen JG, Chang WH, Ballantyne C, et al. Antiinflammatory therapy with canakinumab for atherosclerotic disease. N Engl J Med. 2017;377:1119-31. 
4. Tardif J-C, Kouz S, Waters DD, Bertrand OF, Diaz R, Maggioni AP, et al. Efficacy and safety of low-dose colchicine after myocardial infarction. N Engl J Med. 2019;381:2497-505.

5.• Everett BM, Cornel J, Lainscak M, Anker SD, Abbate A, Thuren T, et al. Anti-Inflammatory therapy with canakinumab for the prevention of hospitalization for heart failure. Circulation. 2019;139: 1289-99 In patients with prior myocardial infarction and elevated high-sensitivity CRP treated with the interleukin-1 $\beta$ monoclonal antibody canakinumab, there was a dosedependent reduction in hospitalisation for heart failure and the composite end-point of hospitalisation for heart failure or heart failure-related mortality.

6. Murphy SP, Kakkar R, McCarthy CP, Januzzi JL. Inflammation in heart failure JACC state-of-the-art review. J Am Coll Cardiol. 2020;75:1324-40.

7. Nahrendorf M, Pittet MJ, Swirski FK. Monocytes: protagonists of infarct inflammation and repair after myocardial infarction. Circulation. 2010;121:2437-45.

8. Westman PC, Lipinski MJ, Luger D, Waksman R, Bonow RO, Wu $\mathrm{E}$, et al. Inflammation as a driver of adverse left ventricular remodeling after acute myocardial infarction. J Am Coll Cardiol. 2016;67: 2050-60.

9. Ismahil MA, Prabhu SD. Cardiac immune cell remodeling after myocardial infarction. J Mol Cell Cardiol. 2013;62:142-3.

10. Dewald O, Zymek P, Winkelmann K, Koerting A, Ren G, AbouKhamis T, et al. CCL2/monocyte chemoattractant protein-1 regulates inflammatory responses critical to healing myocardial infarcts. Circ Res. 2005;96:881-9.

11. Swirski FK, Nahrendorf M, Etzrodt M, Wildgruber M, CortezRetamozo V, Panizzi P, et al. Identification of splenic reservoir monocytes and their deployment to inflammatory sites. Science. 2009;325:612-6.

12. Weirather J, Hofmann UDW, Beyersdorf N, Ramos GC, Vogel B, Frey A, et al. Foxp3+ CD4+ T cells improve healing after myocardial infarction by modulating monocyte/macrophage differentiation. Circ Res. 2014;115:55-67.

13. Nahrendorf M, Swirski FK, Aikawa E, Stangenberg L, Wurdinger $\mathrm{T}$, Figueiredo J-L, et al. The healing myocardium sequentially mobilizes two monocyte subsets with divergent and complementary functions. J Exp Med. 2007;204:3037-47.

14. van der Laan AM, ter Horst EN, Delewi R, Begieneman MPV, Krijnen PAJ, Hirsch A, et al. Monocyte subset accumulation in the human heart following acute myocardial infarction and the role of the spleen as monocyte reservoir. Eur Heart J. 2014;35:376-85.

15. Tsujioka H, Imanishi T, Ikejima H, Kuroi A, Takarada S, Tanimoto $\mathrm{T}$, et al. Impact of heterogeneity of human peripheral blood monocyte subsets on myocardial salvage in patients with primary acute myocardial infarction. J Am Coll Cardiol. 2009;54:130-8.

16. Engström G, Melander O, Hedblad B. Leukocyte count and incidence of hospitalizations due to heart failure. Circ Heart Fail. 2009;2:217-22.

17. Zouggari Y, Ait-Oufella H, Bonnin P, Simon T, Sage AP, Guérin $\mathrm{C}$, et al. B lymphocytes trigger monocyte mobilization and impair heart function after acute myocardial infarction. Nat Med. 2013;19: 1273-80.

18. Sager HB, Hulsmans M, Lavine KJ, Moreira MB, Heidt T, Courties $\mathrm{G}$, et al. Proliferation and recruitment contribute to myocardial macrophage expansion in chronic heart failure. Circ Res. 2016;119:853-64.

19. Majmudar MD, Keliher EJ, Heidt T, Leuschner F, Truelove J, Sena $\mathrm{BF}$, et al. Monocyte-directed RNAi targeting CCR2 improves infarct healing in atherosclerosis-prone mice. Circulation. 2013;127: 2038-46.

20. Leuschner F, Panizzi P, Chico-Calero I, Lee WW, Ueno T, CortezRetamozo V, et al. Angiotensin-converting enzyme inhibition prevents the release of monocytes from their splenic reservoir in mice with myocardial infarction. Circ Res. 2010;107:1364-73.

21. Honold L, Nahrendorf M. Resident and monocyte-derived macrophages in cardiovascular disease. Circ Res. 2018;122:113-27.

22. Dick SA, Macklin JA, Nejat S, Momen A, Clemente-Casares X, Althagafi MG, et al. Self-renewing resident cardiac macrophages limit adverse remodeling following myocardial infarction. Nat Immunol. 2019;20:29-39.

23. Bajpai G, Schneider C, Wong N, Bredemeyer A, Hulsmans M, Nahrendorf M, et al. The human heart contains distinct macrophage subsets with divergent origins and functions. Nat Med. 2018;24: $1234-45$.

24. Bajpai G, Bredemeyer A, Li W, Zaitsev K, Koenig AL, Lokshina I, et al. Tissue resident CCR2- and CCR2+ cardiac macrophages differentially orchestrate monocyte recruitment and fate specification following myocardial injury. Circ Res. 2019;124:263-78.

25. Courties G, Heidt T, Sebas M, Iwamoto Y, Jeon D, Truelove J, et al. In vivo silencing of the transcription factor IRF5 reprograms the macrophage phenotype and improves infarct healing. J Am Coll Cardiol. 2014;63:1556-66.

26. Krishnamurthy P, Rajasingh J, Lambers E, Qin G, Losordo DW, Kishore R. IL-10 inhibits inflammation and attenuates left ventricular remodeling after myocardial infarction via activation of STAT3 and suppression of HuR. Circ Res. 2009;104:e9-18.

27. Nahrendorf M, Swirski FK. Abandoning M1/M2 for a network model of macrophage function. Circ Res. 2016;119:414-7.

28. Peet C, Ivetic A, Bromage DI, Shah AM. Cardiac monocytes and macrophages after myocardial infarction. Cardiovasc Res. 2019;116:1101-12.

29. Hoogeveen RM, Nahrendorf M, Riksen NP, Netea MG, de Winther MPJ, Lutgens E, et al. Monocyte and haematopoietic progenitor reprogramming as common mechanism underlying chronic inflammatory and cardiovascular diseases. Eur Heart J. 2017;39:3521-7.

30. Wan E, Yeap XY, Dehn S, Terry R, Novak M, Zhang S, et al. Enhanced efferocytosis of apoptotic cardiomyocytes through myeloid-epithelial-reproductive tyrosine kinase links acute inflammation resolution to cardiac repair after infarction. Circ Res. 2013;113:1004-12.

31. Lee WW, Marinelli B, van der Laan AM, Sena BF, Gorbatov R, Leuschner $\mathrm{F}$, et al. PET/MRI of inflammation in myocardial infarction. J Am Coll Cardiol. 2012;59:153-63.

32. Wollenweber T, Roentgen P, Schäfer A, Schatka I, Zwadlo C, Brunkhorst $\mathrm{T}$, et al. Characterizing the inflammatory tissue response to acute myocardial infarction by clinical multimodality noninvasive imaging. Circ Cardiovasc Imaging. 2014;7:811-8.

33. Rischpler C, Dirschinger RJ, Nekolla SG, Kossmann H, Nicolosi S, Hanus F, et al. Prospective evaluation of $18 \mathrm{~F}$-fluorodeoxyglucose uptake in postischemic myocardium by simultaneous positron emission tomography/magnetic resonance imaging as a prognostic marker of functional outcome. Circ Cardiovasc Imaging. 2016;9: e004316 A prospective study that compared post-infaret PET inflammatory signals with longitudinal assessment of $\mathbf{L V}$ function. ${ }^{18}$ F-FDG uptake in the first week after myocardial infarction was shown to be associated with an increased likelihood of left ventricular impairment on MRI after 6 months, independent of infarct size.

34. Tarkin JM, Calcagno C, Dweck MR, Evans NR, Chowdhury MM, Gopalan D, et al. 68Ga-DOTATATE PET identifies residual myocardial inflammation and bone marrow activation after myocardial infarction. J Am Coll Cardiol. 2019;73:2489-91.

35. Lapa C, Reiter T, Werner RA, Ertl G, Wester H-J, Buck AK, et al. [68Ga]Pentixafor-PET/CT for imaging of chemokine receptor 4 expression after myocardial infarction. JACC Cardiovasc Imaging. 2015;8:1466-8.

36. Jenkins WSA, Vesey AT, Stirrat C, Connell M, Lucatelli C, Neale A, et al. Cardiac $\alpha$ V $\beta 3$ integrin expression following acute 
myocardial infarction in humans. Heart. 2016;103:607-15 This clinical study highlighted the role of 'reparative' processes such as neoangiogenesis in predicting the likelihood of longer term left ventricular recovery.

37. Siebermair J, Köhler MI, Kupusovic J, Nekolla SG, Kessler L, Ferdinandus J, et al. Cardiac fibroblast activation detected by Ga68 FAPI PET imaging as a potential novel biomarker of cardiac injury/remodeling. J Nucl Cardiol. 2020. https://doi.org/10.1007/ s12350-020-02307-w.

38. Thackeray JT, Hupe HC, Wang Y, Bankstahl JP, Berding G, Ross $\mathrm{TL}$, et al. Myocardial inflammation predicts remodeling and neuroinflammation after myocardial infarction. J Am Coll Cardiol. 2018;71:263-75 Early increases in TSPO PET signals after myocardial infarction in mice were predictive of subsequent adverse left ventricular remodelling at 8 weeks. Treatment with angiotensin-converting enzyme inhibitor reduced the TSPO signal. Interestingly, changes in neuroinflammation as detected by the TSPO PET occurred in parallel to myocardial signal changes.

39. Lapa C, Reiter T, Li X, Werner RA, Samnick S, Jahns R, et al. Imaging of myocardial inflammation with somatostatin receptor based PET/CT - a comparison to cardiac MRI. Int J Cardiol. 2015;194:44-9.

40. Thackeray JT, Derlin T, Haghikia A, Napp LC, Wang Y, Ross TL, et al. Molecular imaging of the chemokine receptor CXCR4 after acute myocardial infarction. JACC Cardiovasc Imaging. 2015;8: 1417-26.

41. Reiter T, Kircher M, Schirbel A, Werner RA, Kropf S, Ertl G, et al. Imaging of $\mathrm{C}-\mathrm{X}-\mathrm{C}$ motif chemokine receptor CXCR4 expression after myocardial infarction with $[68 \mathrm{Ga}]$ Pentixafor-PET/CT in correlation with cardiac MRI. JACC Cardiovasc Imaging. 2018;11: 1541-3.

42. - Hess A, Derlin T, Koenig T, Diekmann J, Wittneben A, Wang Y, et al. Molecular imaging-guided repair after acute myocardial infarction by targeting the chemokine receptor CXCR4. Eur Heart J. 2020;41:3564-75 The incidence of left ventricular rupture in mice after MI was reduced by administration of a CXCR4 blocking agent within the first 3 days post-infarct. In 50 patients, infarct-related CXCR4 signals were inversely correlated with left ventricular function.

43. Heo GS, Bajpai G, Li W, Luehmann HP, Sultan DH, Dun H, et al. Targeted PET imaging of chemokine receptor 2-positive monocytes and macrophages in the injured heart. J Nucl Med. 2020;62: $111-4$.

44. Heo GS, Kopecky B, Sultan D, Ou M, Feng G, Bajpai G, et al. Molecular imaging visualizes recruitment of inflammatory monocytes and macrophages to the injured heart. Circ Res. 2019;124: 881-90.

45. Nahrendorf M, Hoyer FF, Meerwaldt AE, van Leent MMT, Senders ML, Calcagno C, et al. Imaging Cardiovascular and lung macrophages with the positron emission tomography sensor $64 \mathrm{Cu}-$ Macrin in mice, rabbits, and pigs. Circ Cardiovasc Imaging. 2020;13:e010586 In this preclinical study, ${ }^{\mathbf{6 4}} \mathbf{C u}$-Macrin was shown to be taken up by macrophages within infarcted myocardium, with negligible uptake by neutrophils, fibroblasts and endothelial cells.

46. Keliher EJ, Ye Y-X, Wojtkiewicz GR, Aguirre AD, Tricot B, Senders ML, et al. Polyglucose nanoparticles with renal elimination and macrophage avidity facilitate PET imaging in ischaemic heart disease. Nat Commun. 2017;8:14064.

47. Morooka M, Kubota K, Kadowaki H, Ito K, Okazaki O, Kashida $\mathrm{M}$, et al. 11C-Methionine PET of acute myocardial infarction. J Nucl Med. 2009;50:1283-7.

48. Thackeray JT, Bankstahl JP, Wang Y, Wollert KC, Bengel FM. Targeting amino acid metabolism for molecular imaging of inflammation early after myocardial infarction. Theranostics. 2016;6:1768-79.

49. Taki J, Wakabayashi H, Inaki A, Imanaka-Yoshida K, Hiroe M, Ogawa K, et al. 14C-methionine uptake as a potential marker of inflammatory processes after myocardial ischemia and reperfusion. J Nucl Med. 2013;54:431-6.

50. Higuchi T, Bengel FM, Seidl S, Watzlowik P, Kessler H, Hegenloh $\mathrm{R}$, et al. Assessment of $\alpha \mathrm{v} \beta 3$ integrin expression after myocardial infarction by positron emission tomography. Cardiovasc Res. 2008;78:395-403.

51. Sun Y, Zeng Y, Zhu Y, Feng F, Xu W, Wu C, et al. Application of68Ga-PRGD2 PET/CT for $\alpha \mathrm{v} \beta 3$-integrin imaging of myocardial infarction and stroke. Theranostics. 2014;4:778-86.

52. Varasteh Z, Mohanta S, Robu S, Braeuer M, Li Y, Omidvari N, et al. Molecular imaging of fibroblast activity after myocardial infarction using a 68 Ga-labeled fibroblast activation protein inhibitor, FAPI-04. J Nucl Med. 2019;60:1743-9.

53. Heckmann MB, Reinhardt F, Finke D, Katus HA, Haberkorn U, Leuschner F, et al. Relationship between cardiac fibroblast activation protein activity by positron emission tomography and cardiovascular disease. Circ Cardiovasc Imaging. 2020;13:e010628.

54. Ma H, Liu S, Xiong Y, Zhang Z, Sun A, Su S, et al. PET imaging of cardiomyocyte apoptosis in a rat myocardial infarction model. Apoptosis. 2018;23:396-407.

55. Marchesseau S, Seneviratna A, Sjöholm AT, Qin DL, Ho JXM, Hausenloy DJ, et al. Hybrid PET/CT and PET/MRI imaging of vulnerable coronary plaque and myocardial scar tissue in acute myocardial infarction. J Nucl Cardiol. 2018;25:2001-11.

56. Ståhle M, Kytö V, Kiugel M, Liljenbäck H, Metsälä O, Käkelä M, et al. Glucagon-like peptide-1 receptor expression after myocardial infarction: imaging study using 68Ga-NODAGA-exendin-4 positron emission tomography. J Nucl Cardiol. 2020;27:2386-97.

57. Ćorović A, Wall C, Mason JC, Rudd JHF, Tarkin JM. Novel positron emission tomography tracers for imaging vascular inflammation. Curr Cardiol Rep. 2020;22:119. https://doi.org/10.1007/ s11886-020-01372-4.

58. Tarkin JM, Joshi FR, Evans NR, Chowdhury MM, Figg NL, Shah $\mathrm{AV}$, et al. Detection of atherosclerotic inflammation by $68 \mathrm{Ga}-$ DOTATATE PET compared to [18F]FDG PET imaging. J Am Coll Cardiol. 2017;69:1774-91.

59. Tarkin JM, Wall C, Gopalan D, Aloj L, Manavaki R, Fryer TD, et al. Novel approach to imaging active Takayasu arteritis using somatostatin receptor positron emission tomography/magnetic resonance imaging. Circ Cardiovasc Imaging. 2020;13:e010389.

60. Rieckmann M, Delgobo M, Gaal C, Büchner L, Steinau P, Reshef $\mathrm{D}$, et al. Myocardial infarction triggers cardioprotective antigenspecific T helper cell responses. J Clin Invest. 2019;129:4922-36.

61. Wang Y, Dembowsky K, Chevalier E, Stüve P, Korf-Klingebiel M, Lochner $\mathrm{M}$, et al. C-X-C motif chemokine receptor 4 blockade promotes tissue repair after myocardial infarction by enhancing regulatory $\mathrm{T}$ cell mobilization and immune-regulatory function. Circulation. 2019;139:1798-812.

62. Husain M, Birkenfeld AL, Donsmark M, Dungan K, Eliaschewitz FG, Franco DR, et al. Oral semaglutide and cardiovascular outcomes in patients with type 2 diabetes. New Engl J Med. 2019;381:841-51.

63. Autio A, Uotila S, Kiugel M, Kytö V, Liljenbäck H, Kudomi N, et al. $68 \mathrm{Ga}$-DOTA chelate, a novel imaging agent for assessment of myocardial perfusion and infarction detection in a rodent model. $\mathrm{J}$ Nucl Cardiol. 2020;27:891-8.

64. Croteau E, Renaud JM, McDonald M, Klein R, DaSilva JN, Beanlands RSB, et al. Test-retest repeatability of myocardial blood flow and infarct size using $11 \mathrm{C}$-acetate micro-PET imaging in mice. Eur J Nucl Med Mol Imaging. 2015;42:1589-600.

65. Borchert T, Hess A, Lukačević M, Ross TL, Bengel FM, Thackeray JT. Angiotensin-converting enzyme inhibitor treatment early after 
myocardial infarction attenuates acute cardiac and neuroinflammation without effect on chronic neuroinflammation. Eur J Nucl Med Mol I. 2020;47(7):1757-768.

66. Park H, Kim HS, Hong YJ, Min J-J, Kim HB, Kim MC, et al. Therapeutic effect of fimasartan in a rat model of myocardial infarction evaluated by cardiac positron emission tomography with [18F]FPTP. Chonnam Med J. 2019;55:109-15.

67. Li X, Yang W, Ma W, Zhou X, Quan Z, Li G, et al. 18F-FDG PET imaging-monitored anti-inflammatory therapy for acute myocardial infarction: Exploring the role of MCC950 in murine model. J Nucl Cardiol. 2020. https://doi.org/10.1007/s12350-020-02044-0.

68. Hess A, Borchert T, Langer BLN, Wester H, Ross T, Bengel F, et al. Multi-tracer molecular imaging reveals critical role of macrophages for cardiac repair after acute myocardial infarction. J Nucl Med. 2020;61:227 Available from: http://jnm.snmjournals. org/content/61/supplement_1/227.abstract.

69. Kunze KP, Dirschinger RJ, Kossmann H, Hanus F, Ibrahim T, Laugwitz K-L, et al. Quantitative cardiovascular magnetic resonance: extracellular volume, native T1 and 18F-FDG PET/CMR imaging in patients after revascularized myocardial infarction and association with markers of myocardial damage and systemic inflammation. J Cardiovasc Magn Reson. 2018;20:33.

Publisher's Note Springer Nature remains neutral with regard to jurisdictional claims in published maps and institutional affiliations. 